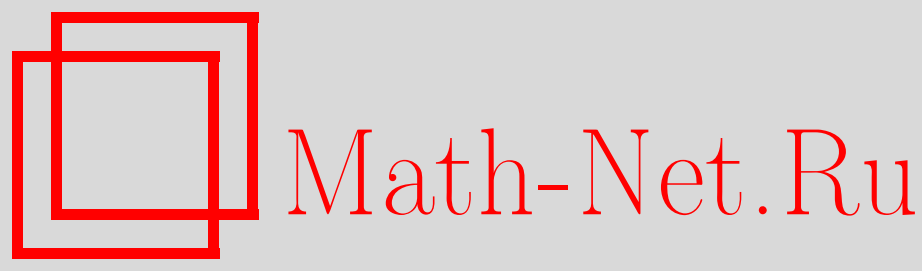

Шу-Гуан Лю, Хун-И Фань, Вывод теоремы о свертке для трехмерного запутанного дробного преобразования Фурье с помощью представления трехчастичного запутанного состояния, ТMФ, 2009, том 161, номер 3, 459-468

DOI: https://doi.org/10.4213/tmf6452

Использование Общероссийского математического портала Math-Net.Ru подразумевает, что вы прочитали и согласны с пользовательским соглашением http://www . mathnet.ru/rus/agreement

Параметры загрузки:

IP : 54.162 .85 .209

26 апреля 2023 г., 15:29:14

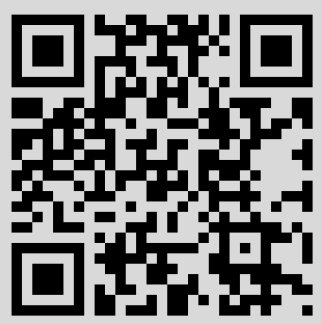




\section{ВЫВОД ТЕОРЕМЫ О СВЕРТКЕ ДЛЯ ТРЕХМЕРНОГО ЗАПУТАННОГО ДРОБНОГО ПРЕОБРАЗОВАНИЯ ФУРЬЕ С ПОМОЩЬЮ ПРЕДСТАВЛЕНИЯ ТРЕХЧАСТИЧНОГО ЗАПУТАННОГО СОСТОЯНИЯ}

Показано, что путем построения двух взаимно сопряженных представлений трехчастичного запутанного состояния можно естественным образом прийти к запутанному преобразованию Фурье. Доказана теорема о свертке для трехмерного запутанного дробного преобразования Фурье в контексте квантовой механики.

Ключевые слова: трехмерное запутанное дробное преобразование Фурье, теорема о свертке, представление трехчастичного запутанного состояния.

\section{1. ВВЕДЕНИЕ}

В последние годы квантовое запутывание и запутанные состояния привлекли большой интерес физиков. Впервые идею квантового запутывания между двумя частицами предложили Эйнштейн, Подольский и Розен [1]. В работах [2], [3] построено представление двухчастичного запутанного состояния, очень полезное для объяснения явления запутывания. Для трехчастичной системы также было выведено представление трехчастичного запутанного состояния [4], [5], и на его основе найдено трехмерное запутанное преобразование Фурье (ЗПФ), отличное от обычного трехмерного преобразования Фурье (ПФ). Другими словами, трехмерное ЗПФ не есть прямое произведение трех одномерных. Для одномерного ПФ Намиас [6] предложил обобщение, называемое дробным преобразованием Фурье (ДПФ). ДПФ и связанные с ним операторы Бозе впервые были использованы в теории открытых резонаторов в шестидесятых годах (см. ссылки в книге [7]) и позднее в работе МакБрайда и Керра [8]. Обычное ДПФ активно использовалось в оптике Фурье и информационной оптике, но не имело большого значения для оптики, пока не было

${ }^{*}$ Department of Applied Mathematics and Physics, Anhui University of Technology and Science, Wuhu, Anhui 241000, China. E-mail: sgliu@mail.ustc.edu.cn

${ }^{\dagger}$ Department of Material Science and Engineering, University of Science and Technology of China, Hefei, Anhui 230026, China 
дано физическое определение ДПФ, основанное на распространении света в квадратичной градиентной среде. В работах [9]-[11] ДПФ дробного порядка $\alpha(\alpha-Д П Ф)$ определено следующим образом: пусть заданная функция вводится с одной стороны квадратичной градиентной среды в точке $z=0$, тогда распределение света, наблюдаемое на плоскости $z=z_{0}$, соответствует $\alpha$-ДПФ введенной функции $\left(\alpha=z_{0} / L\right.$, где $L \equiv(\pi / 2)\left(n_{1} / n_{2}\right)^{1 / 2}$ - характеристическая длина).

Другой подход к введению ДПФ был предложен Лохманом [12], отметившим алгоритмический изоморфизм между поворотом образа, поворотом функции распределения Вигнера и ДПФ. Лохман описал ДПФ как производимое над функцией преобразование, ведущее к повороту ассоциированной функции распределения Вигнера. Для трехмерного ЗПФ можно также предложить трехмерное запутанное дробное преобразование Фурье (ЗДПФ). Цель данной работы - изучить теорему о свертке для трехмерного ЗДПФ. Напомним теорему о свертке ПФ [13].

Если $F_{i}-\Pi \Phi f_{i} \in L^{1}(\mathbb{R}), i=1,2$, то $F_{1} F_{2}-\Pi \Phi\left(f_{1} * f_{2}\right)$, где свертка двух функиий определяется интегралом

$$
\left(f_{1} * f_{2}\right)(x)=\int_{-\infty}^{\infty} f_{1}(y) f_{2}(x-y) d y
$$

или

$$
\int_{-\infty}^{\infty} f_{1}(y) f_{2}(y) e^{-i \omega y} d y=\int_{-\infty}^{\infty} F_{1}(x) F_{2}(\omega-x) d x
$$

В последнее время разные авторы обобщали теорему о свертке ПФ в одномерном случае на дробные области исходя из других подходов [14], [15]. Таким образом, естественно возникает вопрос: каков формализм теоремы о свертке трехмерного ЗДПФ? Можно ли вывести ее в контексте квантовой оптики? Ответ утвердительный. В настоящей работе использовано преобразование между двумя взаимно сопряженными представлениями трехчастичного запутанного состояния для вывода теоремы о свертке. Это связано с тем, что представления запутанных состояний в квантовой механике полные и ортонормированные, а их взаимное преобразование представляет собой просто ЗПФ. Вывод теоремы строг и достаточно краток.

\section{2. КРАТКИЙ ОБЗОР ТРЕХЧАСТИЧНЫХ ЗАПУТАННЫХ СОСТОЯНИЙ}

Одномерное ПФ дробного порядка $\alpha$ определяется так, что обычное ПФ является его особым случаем, т.е.

$$
F_{\alpha}[g(x)]=\frac{1}{(2 \pi \sin \alpha)^{1 / 2}} e^{i(\alpha-\pi / 2) / 2} \int_{-\infty}^{\infty} \exp \left[\frac{i\left(x^{2}+p^{2}\right)}{2 \operatorname{tg} \alpha}-\frac{i x p}{\sin \alpha}\right] g(x) d x \equiv G(p),
$$

где экспоненциальная функция - ядро интеграла. Это преобразование можно рассматривать как особое преобразование координатно-импульсного представления в контексте квантовой механики [16]. Для трехчастичного случая в работах [4], [5] построено два взаимно сопряженных трехмодовых запутанных состояния $\left|p, \chi_{2}, \chi_{3}\right\rangle$ 
и $\left|\chi, p_{2}, p_{3}\right\rangle$, где

$$
\begin{aligned}
\left|p, \chi_{2}, \chi_{3}\right\rangle= & \frac{1}{\sqrt{3} \pi^{3 / 4}} \exp \left[-\frac{p^{2}}{6}-\frac{1}{3}\left(\chi_{2}^{2}+\chi_{3}^{2}-\chi_{2} \chi_{3}\right)+\frac{\sqrt{2} i p}{3} \sum_{i=1}^{3} a_{i}^{\dagger}+\right. \\
& +\frac{\sqrt{2} \chi_{2}}{3}\left(a_{1}^{\dagger}-2 a_{2}^{\dagger}+a_{3}^{\dagger}\right)+\frac{\sqrt{2} \chi_{3}}{3}\left(a_{1}^{\dagger}+a_{2}^{\dagger}-2 a_{3}^{\dagger}\right)+ \\
& \left.+\frac{2}{3} \sum_{j>i=1}^{3} a_{i}^{\dagger} a_{j}^{\dagger}-\frac{1}{6} \sum_{i=1}^{3} a_{i}^{\dagger 2}\right]|000\rangle,
\end{aligned}
$$

что является общим собственным вектором трех совместимых операторов $X_{1 j}=$ $X_{1}-X_{j}, j=2,3$, относительных координат двух частиц и оператора полного импульса $P=\sum_{i=1}^{3} P_{i}$, т.е.

$$
\begin{aligned}
X_{1 j}\left|p, \chi_{2}, \chi_{3}\right\rangle & =\chi_{j}\left|p, \chi_{2}, \chi_{3}\right\rangle, \quad j=2,3, \\
P\left|p, \chi_{2}, \chi_{3}\right\rangle & =p\left|p, \chi_{2}, \chi_{3}\right\rangle .
\end{aligned}
$$

Здесь $a_{i}^{\dagger}, a_{i}, i=1,2,3,-$ операторы Бозе, удовлетворяющие соотношению $\left[a_{i}, a_{i}^{\dagger}\right]=1$, они связаны с $X_{i}, P_{i}$ соотношениями

$$
X_{i}=\frac{1}{\sqrt{2}}\left(a_{i}+a_{i}^{\dagger}\right), \quad P_{i}=\frac{1}{\sqrt{2} i}\left(a_{i}-a_{i}^{\dagger}\right) .
$$

Сопряженное состояние $\left|p, \chi_{2}, \chi_{3}\right\rangle$ определяется выражением

$$
\begin{aligned}
\left|\chi, p_{2}, p_{3}\right\rangle= & \frac{1}{\sqrt{3} \pi^{3 / 4}} \exp \left[-\frac{\chi^{2}}{6}-\frac{1}{3}\left(p_{2}^{2}+p_{3}^{2}-p_{2} p_{3}\right)+\frac{\sqrt{2} \chi}{3} \sum_{i=1}^{3} a_{i}^{\dagger}+\right. \\
& +\frac{i \sqrt{2} p_{2}}{3}\left(a_{1}^{\dagger}-2 a_{2}^{\dagger}+a_{3}^{\dagger}\right)+\frac{i \sqrt{2} p_{3}}{3}\left(a_{1}^{\dagger}+a_{2}^{\dagger}-2 a_{3}^{\dagger}\right)- \\
& \left.-\frac{2}{3} \sum_{j>i=1}^{3} a_{i}^{\dagger} a_{j}^{\dagger}+\frac{1}{6} \sum_{i=1}^{3} a_{i}^{\dagger 2}\right]|000\rangle,
\end{aligned}
$$

что является общим собственным вектором операторов $P_{1 j}=P_{1}-P_{j}, j=2,3$, и $X=\sum_{i=1}^{3} X_{i}$,

$$
\begin{aligned}
X\left|\chi, p_{2}, p_{3}\right\rangle & =\chi\left|\chi, p_{2}, p_{3}\right\rangle, \\
P_{1 j}\left|\chi, p_{2}, p_{3}\right\rangle & =P_{j}\left|\chi, p_{2}, p_{3}\right\rangle, \quad j=2,3 .
\end{aligned}
$$

Заметим, что

$$
\frac{1}{2}\left[X_{1 j}, P_{1 j}\right]=i, \quad \frac{1}{3}[X, P]=i,
$$

$X_{1 j}$ сопряжен к $P_{1 j}, X$ сопряжен к $P$, так что $\left|p, \chi_{2}, \chi_{3}\right\rangle$ сопряжен к $\left|\chi, p_{2}, p_{3}\right\rangle$. Оба полны и ортонормированны,

$$
\begin{gathered}
\int_{-\infty}^{\infty} d p d \chi_{2} d \chi_{3}\left|p, \chi_{2}, \chi_{3}\right\rangle\left\langle p, \chi_{2}, \chi_{3}\right|=1 \\
\left\langle p, \chi_{2}, \chi_{3} \mid p^{\prime}, \chi_{2}^{\prime}, \chi_{3}^{\prime}\right\rangle=\delta\left(p-p^{\prime}\right) \delta\left(\chi_{2}-\chi_{2}^{\prime}\right) \delta\left(\chi_{3}-\chi_{3}^{\prime}\right) \\
\int_{-\infty}^{\infty} d \chi d p_{2} d p_{3}\left|\chi, p_{2}, p_{3}\right\rangle\left\langle\chi, p_{2}, p_{3}\right|=1 \\
\left\langle\chi, p_{2}, p_{3} \mid \chi^{\prime}, p_{2}^{\prime}, p_{3}^{\prime}\right\rangle=\delta\left(\chi-\chi^{\prime}\right) \delta\left(p_{2}-p_{2}^{\prime}\right) \delta\left(p_{3}-p_{3}^{\prime}\right) .
\end{gathered}
$$


Пересечение между ними имеет вид

$$
\left\langle p, \chi_{2}, \chi_{3} \mid \chi, p_{2}, p_{3}\right\rangle=\frac{1}{3(2 \pi)^{3 / 2}} \exp \left\{-\frac{i}{3}\left(p \chi+\chi_{2} p_{3}+\chi_{3} p_{2}\right)+\frac{2 i}{3}\left(p_{2} \chi_{2}+p_{3} \chi_{3}\right)\right\},
$$

что можно подтвердить следующим интегрированием с использованием выражений (4) и (7):

$$
\begin{aligned}
& \frac{1}{3(2 \pi)^{3 / 2}} \int_{-\infty}^{\infty} d p d \chi_{2} d \chi_{3}\left|p, \chi_{2}, \chi_{3}\right\rangle \exp \left\{-\frac{i}{3}\left(p \chi+\chi_{2} p_{3}+\chi_{3} p_{2}\right)+\frac{2 i}{3}\left(p_{2} \chi_{2}+p_{3} \chi_{3}\right)\right\}= \\
& \quad=\left|\chi, p_{2}, p_{3}\right\rangle .
\end{aligned}
$$

Сравнив выражение (10) с традиционным трехмерным преобразованием координатно-импульсного представления

$$
\frac{1}{(2 \pi)^{3 / 2}} e^{-i \vec{p} \cdot \vec{x}}=\langle\vec{p} \mid \vec{x}\rangle, \quad \vec{x}=\left(x_{1}, x_{2}, x_{3}\right), \quad \vec{p}=\left(p_{1}, p_{2}, p_{3}\right),
$$

можно заключить, что преобразование представления в (10) происходит запутанным образом. Это ясно из следующего наблюдения. Если мы положим

$$
y_{1}=\frac{\chi}{3}, \quad y_{2}=\frac{-2 p_{2}+p_{3}}{3}, \quad y_{3}=\frac{p_{2}-2 p_{3}}{3}
$$

в выражении (11), то оно примет вид

$$
\begin{aligned}
& \frac{1}{3(2 \pi)^{3 / 2}} \int_{-\infty}^{\infty} d p d \chi_{2} d \chi_{3}\left|p, \chi_{2}, \chi_{3}\right\rangle e^{-i\left(y_{1} p+y_{2} \chi_{2}+y_{3} \chi_{3}\right)}= \\
& \quad=\left|3 y_{1},-\left(2 y_{2}+y_{3}\right),-\left(2 y_{3}+y_{2}\right)\right\rangle .
\end{aligned}
$$

Хотя левая часть кажется нормальным ПФ ( $y, y_{2}, y_{3}$ независимы друг от друга), правая часть дает функцию от $-\left(2 y_{2}+y_{3}\right)$ и $-\left(2 y_{3}+y_{2}\right)$. Другими словами, когда мы имеем дело с преобразованием представления для двух взаимно сопряженных запутанных состояний, неминуемо приходится производить преобразование запутанным образом, так что мы называем преобразование

$$
\begin{gathered}
\frac{1}{3(2 \pi)^{3 / 2}} \int_{-\infty}^{\infty} d p d p_{2} d p_{3} g\left(\chi, p_{2}, p_{3}\right) \exp \left\{-\frac{i}{3}\left(p \chi+\chi_{2} p_{3}+\chi_{3} p_{2}\right)+\frac{2 i}{3}\left(p_{2} \chi_{2}+p_{3} \chi_{3}\right)\right\}= \\
=\int_{-\infty}^{\infty} d \chi d p_{2} d p_{3}\left\langle p, \chi_{2}, \chi_{3} \mid \chi, p_{2}, p_{3}\right\rangle\left\langle\chi, p_{2}, p_{3} \mid g\right\rangle=\left\langle p, \chi_{2}, \chi_{3} \mid g\right\rangle
\end{gathered}
$$

трехмерным ЗПФ, где $g\left(\chi, p_{2}, p_{3}\right)$ - волновая функция $|g\rangle$ в $\left\langle\chi, p_{2}, p_{3}\right|$-представлении, $g\left(\chi, p_{2}, p_{3}\right)=\left\langle\chi, p_{2}, p_{3} \mid g\right\rangle$, и волновая функция состояния $|g\rangle$ в $\left\langle\chi, p_{2}, p_{3}\right|$-представлении превращается в волновую функцию $|g\rangle$ в $\left\langle p, \chi_{2}, \chi_{3}\right|$-представлении. Очевидно, оно отличается от обычного трехмерного ПФ.

\section{3. ТРЕХМЕРНОЕ ЗДПФ}

Напомнив, что одномерное ДПФ - это просто матричный элемент

$$
\left\langle p\left|\exp \left[i\left(\frac{\pi}{2}-\alpha\right)\left(a_{1}^{\dagger} a_{1}+\frac{1}{2}\right)\right]\right| x\right\rangle
$$


где $\langle p|$ - одномодовое собственное состояние импульса, $|x\rangle$ - одномодовое собственное состояние координаты, мы предполагаем, что ядро трехмерного ЗДПФ представляет собой просто следующий матричный элемент:

$$
\begin{gathered}
\left\langle p, \chi_{2}, \chi_{3}\left|e^{O}\right| \chi, p_{2}, p_{3}\right\rangle \\
e^{O} \equiv \exp \left[i\left(\frac{\pi}{2}-\alpha\right)_{i=1}^{3}\left(a_{i}^{\dagger} a_{i}+\frac{1}{2}\right)\right] .
\end{gathered}
$$

Основываясь на преобразовании (15) и используя соотношения (4) и (7), вычислим матричный элемент (16). Введем операторы

$$
\begin{aligned}
J_{+} & =\frac{1}{12} \sum_{i=1}^{3}\left(a_{i}^{2}+a_{i}^{\dagger 2}\right)-\frac{1}{3} \sum_{j>i=1}^{3}\left(a_{i} a_{j}+a_{i}^{\dagger} a_{j}^{\dagger}\right)+\frac{1}{2} \sum_{i=1}^{3}\left(a_{i}^{\dagger} a_{i}+\frac{1}{2}\right)= \\
& =\frac{1}{6}\left[P^{2}+2\left(X_{12}^{2}-X_{12} X_{13}+X_{13}^{2}\right)\right], \\
J_{-} & =-\frac{1}{12} \sum_{i=1}^{3}\left(a_{i}^{2}+a_{i}^{\dagger 2}\right)+\frac{1}{3} \sum_{j>i=1}^{3}\left(a_{i} a_{j}+a_{i}^{\dagger} a_{j}^{\dagger}\right)+\frac{1}{2} \sum_{i=1}^{3}\left(a_{i}^{\dagger} a_{i}+\frac{1}{2}\right)= \\
& =\frac{1}{6}\left[X^{2}+2\left(P_{12}^{2}-P_{12} P_{13}+P_{13}^{2}\right)\right] .
\end{aligned}
$$

Мы видим, что

$$
\left[J_{-}, J_{+}\right]=-2 J_{0}
$$

где

$$
J_{0}=\frac{1}{12} \sum_{i=1}^{3}\left(a_{i}^{2}-a_{i}^{\dagger 2}\right)+\frac{1}{3} \sum_{j>i=1}^{3}\left(a_{i}^{\dagger} a_{j}^{\dagger}-a_{i} a_{j}\right)
$$

Очевидно,

$$
J_{+}+J_{-}=\sum_{i=1}^{3}\left(a_{i}^{\dagger} a_{i}+\frac{1}{2}\right) .
$$

С учетом равенства $\left[a_{i}, a_{j}^{\dagger}\right]=\delta_{i j}$ получаем отношение замыкания

$$
\left[J_{-}, J_{0}\right]=J_{-}, \quad\left[J_{+}, J_{0}\right]=-J_{+} .
$$

Заметим, что операторы $J_{+}$и $J_{-}$не являются взаимно эрмитово сопряженными, поэтому $J_{+}, J_{-}, J_{0}$ составляют новую неунитарную бозонную реализацию Ли алгебры $S U(2)$ в трехмодовом пространстве Фока.

Напомним формулу распутывания оператора преобразованием смежных классов $S U(2)[17]$ :

$$
e^{\zeta J_{+}-\zeta^{*} J_{-}}=e^{\tau J_{+}} e^{J_{0} \ln \left(1+|\tau|^{2}\right)} e^{-\tau^{*} J_{-}},
$$

где $\zeta=\theta e^{-i \varphi}, \tau=e^{-i \varphi} \operatorname{tg} \theta$ и $1+|\tau|^{2}=\sec ^{2} \theta$, так что если $\zeta-$ чисто мнимое число, $\zeta=i \theta=\theta e^{i \pi / 2}, \tau=i \operatorname{tg} \theta$, формула (21) сводится к

$$
e^{i \theta\left(J_{+}+J_{-}\right)}=e^{i J_{+} \operatorname{tg} \theta} e^{J_{0} \ln \sec ^{2} \theta} e^{i J_{-} \operatorname{tg} \theta} .
$$


Отсюда с учетом выражений (17), (18) имеем

$$
\begin{aligned}
& \exp \left[i \theta \sum_{i=1}^{3}\left(a_{i}^{\dagger} a_{i}+\frac{1}{2}\right)\right]=e^{i \theta\left(J_{+}+J_{-}\right)}= \\
& =\exp \left\{\frac{i}{6}\left[P^{2}+2\left(X_{12}^{2}-X_{12} X_{13}+X_{13}^{2}\right)\right] \operatorname{tg} \theta\right\} \times \\
& \times \exp \left\{\left[\frac{1}{12} \sum_{i=1}^{3}\left(a_{i}^{2}-a_{i}^{\dagger 2}\right)+\frac{1}{3} \sum_{j>i=1}^{3}\left(a_{i}^{\dagger} a_{j}^{\dagger}-a_{i} a_{j}\right)\right] \ln \sec ^{2} \theta\right\} \times \\
& \times \exp \left\{\frac{i}{6}\left[X^{2}+2\left(P_{12}^{2}-P_{12} P_{13}+P_{13}^{2}\right)\right] \operatorname{tg} \theta\right\} \text {. }
\end{aligned}
$$

Согласно формулам (10) и (23), а также с учетом выражений (5) и (8) для собственных векторов получаем следующий матричный элемент:

$$
\begin{aligned}
&\left\langle p, \chi_{2}, \chi_{3}\left|\exp \left[i \theta \sum_{i=1}^{3}\left(a_{i}^{\dagger} a_{i}+\frac{1}{2}\right)\right]\right| \chi, p_{2}, p_{3}\right\rangle= \\
&=\exp \left\{i \operatorname{tg} \theta \frac{\chi^{2}+2 p_{2}^{2}+2 p_{3}^{2}-2 p_{2} p_{3}+p^{2}+2 \chi_{2}^{2}+2 \chi_{3}^{2}-2 \chi_{2} \chi_{3}}{6}\right\} \times \\
& \quad \times\left\langle p, \chi_{2}, \chi_{3}\left|e^{2 J_{0} \ln \sec \theta}\right| \chi, p_{2}, p_{3}\right\rangle .
\end{aligned}
$$

Заметим, что выражение для $J_{0}(18)$ можно разбить на две части:

$$
2 J_{0}=W-W^{\dagger}, \quad e^{2 \lambda J_{0}}=e^{\lambda\left(W-W^{\dagger}\right)},
$$

где

$$
W^{\dagger}=\frac{1}{6} \sum_{i=1}^{3} a_{i}^{\dagger 2}-\frac{2}{3} \sum_{j>i=1}^{3} a_{i}^{\dagger} a_{j}^{\dagger}
$$

так что $e^{\lambda\left(W_{3}-W_{3}^{\dagger}\right)}-$ трехмодовый сжимающий оператор. Как показано в работе [4], он действует следующим образом:

$$
e^{\lambda\left(W_{3}-W_{3}^{\dagger}\right)}\left|\chi, p_{2}, p_{3}\right\rangle=\mu^{3 / 2}\left|\mu \chi, \mu p_{2}, \mu p_{3}\right\rangle,
$$

$\mu=e^{\lambda}-$ параметр сжатия. Таким образом, имеем

$$
e^{2 J_{0} \ln \sec \theta}\left|\chi, p_{2}, p_{3}\right\rangle=(\sec \theta)^{3 / 2}\left|\chi \sec \theta, p_{2} \sec \theta, p_{3} \sec \theta\right\rangle
$$

Используя формулы (10) и (26), получаем

$$
\begin{aligned}
& \left\langle p, \chi_{2}, \chi_{3}\left|e^{2 J_{0} \ln \sec \theta}\right| \chi, p_{2}, p_{3}\right\rangle= \\
& \quad=\frac{1}{3(2 \pi \cos \theta)^{3 / 2}} \exp \left\{-\frac{i}{3 \cos \theta}\left(p \chi+p_{2} \chi_{3}+\chi_{2} p_{3}-2 p_{2} \chi_{2}-2 p_{3} \chi_{3}\right)\right\} .
\end{aligned}
$$


Подставляя выражение $(27)$ в (24) и положив $\theta \rightarrow \pi / 2-\alpha$, выводим

$$
\begin{aligned}
\left\langle p, \chi_{2}, \chi_{3}\left|e^{O}\right| \chi, p_{2}, p_{3}\right\rangle= & \\
= & \frac{1}{3(2 \pi \sin \alpha)^{3 / 2}} \exp \left\{\frac{i}{6 \operatorname{tg} \alpha}\left(\chi^{2}+2 p_{2}^{2}-2 p_{2} p_{3}+2 p_{3}^{2}+p^{2}+2 \chi_{2}^{2}-2 \chi_{2} \chi_{3}+2 \chi_{3}^{2}\right)-\right. \\
& \left.-\frac{i}{3 \sin \alpha}\left(p \chi+p_{2} \chi_{3}+\chi_{2} p_{3}-2 p_{2} \chi_{2}-2 p_{3} \chi_{3}\right)\right\} \equiv A(\alpha) .
\end{aligned}
$$

Таким образом, вычисление матричного элемента (16) завершено. Взяв $A(\alpha)$ за ядро преобразования, можно определить следующее $\alpha$-ЗДПФ функции $g\left(\chi, p_{2}, p_{3}\right)$ :

$$
\mathfrak{F}_{\alpha}\left[g\left(\chi, p_{2}, p_{3}\right)\right]=e^{i 3(\alpha-\pi / 2) / 2} \int_{-\infty}^{\infty} d \chi d p_{2} d p_{3} A(\alpha) g\left(\chi, p_{2}, p_{3}\right) .
$$

Из формулы (28) и отношения полноты (9) видим, что на самом деле ЗДПФ $\left\langle\chi, p_{2}, p_{3} \mid g\right\rangle$ можно записать в виде

$$
\begin{aligned}
& \mathfrak{F}_{\alpha}\left[g\left(\chi, p_{2}, p_{3}\right)\right]= \\
& \quad=e^{i 3(\alpha-\pi / 2) / 2} \int_{-\infty}^{\infty} d \chi d p_{2} d p_{3}\left\langle p, \chi_{2}, \chi_{3}\left|e^{O}\right| \chi, p_{2}, p_{3}\right\rangle\left\langle\chi, p_{2}, p_{3} \mid g\right\rangle= \\
& \quad=\left\langle p, \chi_{2}, \chi_{3}\left|\exp \left[i\left(\frac{\pi}{2}-\alpha\right) \sum_{i=1}^{3} a_{i}^{\dagger} a_{i}\right]\right| g\right\rangle \equiv G\left(p, \chi_{2}, \chi_{3}\right) .
\end{aligned}
$$

Далее, пусть $G\left(p, \chi_{2}, \chi_{3}\right) \equiv\left\langle p, \chi_{2}, \chi_{3} \mid G\right\rangle ;$ из (30) следует

$$
|G\rangle=e^{-i 3(\alpha-\pi / 2) / 2} e^{O}|g\rangle \equiv|g\rangle \text {. }
$$

Таким образом, $|G\rangle$ - преобразованное с помощью ЗДПФ состояние $|g\rangle$, и $e^{O}$ называется оператором ЗДПФ. Тем самым ЗДПФ $g\left(\chi, p_{2}, p_{3}\right)$ было переформулировано в контексте квантовой механики, что может помочь легко вычислять ЗДПФ квантово-механических волновых функций.

\section{4. ТЕОРЕМА О СВЕРТКЕ ДЛЯ ТРЕХМЕРНОГО ЗДПФ}

Теперь мы сформулируем теорему о свертке для ЗДПФ, продолжающую теорему о свертке для ПФ.

ТЕорема. Пусть $\mathfrak{F}_{\alpha}\left[f\left(\chi, p_{2}, p_{3}\right)\right]\left(p, \chi_{2}, \chi_{3}\right) \equiv \mathfrak{F}_{\alpha}\left(p, \chi_{2}, \chi_{3}\right)-$ ЗДПФ функиии $f\left(\chi, p_{2}, p_{3}\right) u \mathcal{G}_{\alpha}\left[g\left(\chi, p_{2}, p_{3}\right)\right]\left(p, \chi_{2}, \chi_{3}\right) \equiv \mathcal{G}_{\alpha}\left(p, \chi_{2}, \chi_{3}\right)-$ ЗДПФ функиии $g\left(\chi, p_{2}, p_{3}\right)$.

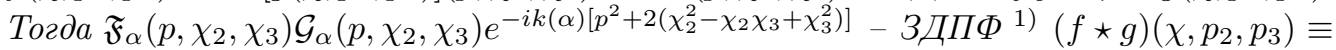
$h\left(\chi, p_{2}, p_{3}\right)$, где свертка двух функиий в смысле ЗДПФ определяется как

$$
(f \star g)\left(\chi, p_{2}, p_{3}\right)=\frac{1}{3(2 \pi \sin \alpha)^{3 / 2}} e^{-i k(\alpha) \Upsilon}(\tilde{f} * \tilde{g})\left(\chi, p_{2}, p_{3}\right) \equiv h\left(\chi, p_{2}, p_{3}\right),
$$

$u$

$$
(\tilde{f} * \tilde{g})\left(\chi, p_{2}, p_{3}\right)=\int d \beta d \gamma d \omega \tilde{f}(\beta, \gamma, \omega) \tilde{g}\left(\chi-\beta, p_{2}-\gamma, p_{3}-\omega\right)
$$

1) Здесь $\star$ обозначает операцию свертки для ЗДПФ, отличную от * в (1). 
где

$$
\begin{gathered}
\tilde{f}\left(\chi, p_{2}, p_{3}\right)=f\left(\chi, p_{2}, p_{3}\right) e^{i k(\alpha) \Upsilon}, \\
\Upsilon=\chi^{2}+2\left(p_{2}^{2}-p_{2} p_{3}+p_{3}^{2}\right), \quad k(\alpha)=\frac{1}{6 \operatorname{tg} \alpha} .
\end{gathered}
$$

Это утверждение можно сформулировать так: ЗДПФ функиии $h\left(\chi, p_{2}, p_{3}\right)-$ это $H_{\alpha}\left(p, \chi_{2}, \chi_{3}\right)$,

$$
\begin{aligned}
\mathfrak{F}_{\alpha}\left[h\left(\chi, p_{2}, p_{3}\right)\right] & =e^{i 3(\alpha-\pi / 2) / 2} \int d \chi d p_{2} d p_{3} A(\alpha) h\left(\chi, p_{2}, p_{3}\right)= \\
& =e^{i 3(\alpha-\pi / 2) / 2} \int d \chi d p_{2} d p_{3}\left\langle p, \chi_{2}, \chi_{3}\left|e^{O}\right| \chi, p_{2}, p_{3}\right\rangle\left\langle\chi, p_{2}, p_{3} \mid h\right\rangle= \\
& =\mathfrak{F}_{\alpha}\left(p, \chi_{2}, \chi_{3}\right) \mathcal{G}_{\alpha}\left(p, \chi_{2}, \chi_{3}\right) e^{-i k(\alpha)\left[p^{2}+2\left(\chi_{2}^{2}-\chi_{2} \chi_{3}+\chi_{3}^{2}\right)\right]} \equiv \\
& \equiv H_{\alpha}\left(p, \chi_{2}, \chi_{3}\right) .
\end{aligned}
$$

ДокАЗАТЕЛьство. Для доказательства этой теоремы мы используем дираковские обозначения бра- и кет-состояний, вводя

$$
\begin{gathered}
h\left(\chi, p_{2}, p_{3}\right)=\left\langle\chi, p_{2}, p_{3} \mid h\right\rangle=\left\langle\chi, p_{2}, p_{3} \mid f \star g\right\rangle, \\
(\tilde{f} * \tilde{g})\left(\chi, p_{2}, p_{3}\right)=\left\langle\chi, p_{2}, p_{3} \mid \tilde{f} * \tilde{g}\right\rangle .
\end{gathered}
$$

Тогда согласно (30), (31) и (9), а также (32) имеем

$$
\begin{aligned}
H_{\alpha}\left(p, \chi_{2}, \chi_{3}\right)= & \left\langle p, \chi_{2}, \chi_{3} \mid H\right\rangle=B\left\langle p, \chi_{2}, \chi_{3}\left|e^{O}\right| h\right\rangle= \\
= & B \int d \chi d p_{2} d p_{3}\left\langle p, \chi_{2}, \chi_{3}\left|e^{O}\right| \chi, p_{2}, p_{3}\right\rangle\left\langle\chi, p_{2}, p_{3} \mid f \star g\right\rangle= \\
= & \frac{B}{3 \Omega^{3 / 2}} \int d \chi d p_{2} d p_{3}\left\langle p, \chi_{2}, \chi_{3}\left|e^{O}\right| \chi, p_{2}, p_{3}\right\rangle \times \\
& \quad \times\left\{\left\langle\chi, p_{2}, p_{3} \mid \tilde{f} * \tilde{g}\right\rangle e^{-i k(\alpha) \Upsilon}\right\},
\end{aligned}
$$

где $B=e^{-i 3(\alpha-\pi / 2) / 2}, \Omega=2 \pi \sin \alpha$. Из (33) и (34) получаем

$$
\begin{aligned}
\left\langle\chi, p_{2}, p_{3} \mid \tilde{f} * \tilde{g}\right\rangle= & \int d \beta d \gamma d \omega \tilde{f}(\beta, \gamma, \omega) \tilde{g}\left(\chi-\beta, p_{2}-\gamma, p_{3}-\omega\right)= \\
= & \int d \beta d \gamma d \omega\langle\beta, \gamma, \omega \mid f\rangle e^{i k(\alpha)\left[\beta^{2}+2\left(\gamma^{2}-\gamma \omega+\omega^{2}\right)\right]}\left\langle\chi-\beta, p_{2}-\gamma, p_{3}-\omega \mid g\right\rangle \times \\
& \times e^{i k(\alpha)\left[(\chi-\beta)^{2}+2\left(p_{2}-\gamma\right)^{2}-2\left(p_{2}-\gamma\right)\left(p_{3}-\omega\right)+2\left(p_{3}-\omega\right)^{2}\right]},
\end{aligned}
$$

где вектор состояния $\langle\beta, \gamma, \omega|$ принадлежит множеству $\left\langle\chi, p_{2}, p_{3}\right|$, т.е. $\langle\beta, \gamma, \omega|$ обозначает $\left\langle\chi=\beta, p_{2}=\gamma, p_{3}=\omega\right|$, так что соотношение (37) принимает вид

$$
\begin{aligned}
H_{\alpha}\left(p, \chi_{2}, \chi_{3}\right)= & \frac{B}{3 \Omega^{3 / 2}} \int d \chi d p_{2} d p_{3}\left\langle p, \chi_{2}, \chi_{3}\left|e^{O}\right| \chi, p_{2}, p_{3}\right\rangle e^{-i k(\alpha) \Upsilon} \times \\
& \times \int d \beta d \gamma d \omega\langle\beta, \gamma, \omega \mid f\rangle e^{i k(\alpha)\left[\beta^{2}+2\left(\gamma^{2}-\gamma \omega+\omega^{2}\right)\right]} \times \\
& \times\left\langle\chi-\beta, p_{2}-\gamma, p_{3}-\omega \mid g\right\rangle \times
\end{aligned}
$$




$$
\begin{aligned}
& \times e^{i k(\alpha)\left[(\chi-\beta)^{2}+2\left(p_{2}-\gamma\right)^{2}-2\left(p_{2}-\gamma\right)\left(p_{3}-\omega\right)+2\left(p_{3}-\omega\right)^{2}\right]}= \\
= & \frac{B}{3 \Omega^{3 / 2}} \int d \chi d p_{2} d p_{3} d \beta d \gamma d \omega\left\langle p, \chi_{2}, \chi_{3}\left|e^{O}\right| \chi+\beta, p_{2}+\gamma, p_{3}+\omega\right\rangle \times \\
& \times\langle\beta, \gamma, \omega \mid f\rangle\left\langle\chi, p_{2}, p_{3} \mid g\right\rangle e^{-2 i k(\alpha)\left(\beta \chi+2 \gamma p_{2}+2 \omega p_{3}-\omega p_{2}-\gamma p_{3}\right)},
\end{aligned}
$$

где

$$
\begin{aligned}
& \frac{B}{3 \Omega^{3 / 2}}\left\langle p, \chi_{2}, \chi_{3}\left|e^{O}\right| \chi+\beta, p_{2}+\gamma, p_{3}+\omega\right\rangle e^{-2 i k(\alpha)\left(\beta \chi+2 \gamma p_{2}+2 \omega p_{3}-\omega p_{2}-\gamma p_{3}\right)}= \\
&=\frac{1}{3 \Omega^{3 / 2}} \exp \left\{\frac{i}{6 \operatorname{tg} \alpha}\left(p^{2}+2 \chi_{2}^{2}-2 \chi_{2} \chi_{3}+2 \chi_{3}^{2}+\beta^{2}+2 \gamma^{2}-2 \gamma \omega+2 \omega^{2}\right)-\right. \\
&\left.\quad-\frac{i}{3 \sin \alpha}\left(\beta p-2 \gamma \chi_{2}-2 \omega \chi_{3}+\omega \chi_{2}+\gamma \chi_{3}\right)\right\} \times \\
& \quad \times \frac{1}{3 \Omega^{3 / 2}} \exp \left\{\frac{i}{6 \operatorname{tg} \alpha}\left(p^{2}+2 p_{2}^{2}-2 p_{2} p_{3}+2 p_{3}^{2}+\chi^{2}+2 \chi_{2}^{2}-2 \chi_{2} \chi_{3}+2 \chi_{3}^{2}\right)-\right. \\
&\left.-\frac{i}{3 \sin \alpha}\left(\chi p-2 p_{2} \chi_{2}-2 \gamma \chi_{2}+p_{3} \chi_{2}+p_{2} \chi_{3}\right)\right\} e^{-i k(\alpha)\left[p^{2}+2\left(\chi_{2}^{2}-\chi_{2} \chi_{3}+\chi_{3}^{2}\right)\right]}= \\
&= B^{2}\left\langle p, \chi_{2}, \chi_{3}\left|e^{O}\right| \beta, \gamma, \omega\right\rangle\left\langle p, \chi_{2}, \chi_{3}\left|e^{O}\right| \chi, p_{2}, p_{3}\right\rangle e^{-i k(\alpha)\left[p^{2}+2\left(\chi_{2}^{2}-\chi_{2} \chi_{3}+\chi_{3}^{2}\right)\right]}
\end{aligned}
$$

Подставляя (40) в (39), имеем

$$
\begin{aligned}
H_{\alpha}\left(p, \chi_{2}, \chi_{3}\right)= & B^{2} \int d \chi d p_{2} d p_{3} d \beta d \gamma d \omega\left\langle p, \chi_{2}, \chi_{3}\left|e^{O}\right| \beta, \gamma, \omega\right\rangle\langle\beta, \gamma, \omega \mid f\rangle \times \\
& \times\left\langle p, \chi_{2}, \chi_{3}\left|e^{O}\right| \chi, p_{2}, p_{3}\right\rangle\left\langle\chi, p_{2}, p_{3} \mid g\right\rangle e^{-i k(\alpha)\left[p^{2}+2\left(\chi_{2}^{2}-\chi_{2} \chi_{3}+\chi_{3}^{2}\right)\right]}= \\
= & B^{2}\left\langle p, \chi_{2}, \chi_{3}\left|e^{O}\right| f\right\rangle\left\langle p, \chi_{2}, \chi_{3}\left|e^{O}\right| g\right\rangle e^{-i k(\alpha)\left[p^{2}+2\left(\chi_{2}^{2}-\chi_{2} \chi_{3}+\chi_{3}^{2}\right)\right]},
\end{aligned}
$$

а это просто символ Дирака для $\mathfrak{F}_{\alpha}\left(p, \chi_{2}, \chi_{3}\right) \mathcal{G}_{\alpha}\left(p, \chi_{2}, \chi_{3}\right) e^{-i k(\alpha)\left[p^{2}+2\left(\chi_{2}^{2}-\chi_{2} \chi_{3}+\chi_{3}^{2}\right)\right]}$. Таким образом, мы получили равенство (35), что завершает доказательство.

Обратное преобразование выражения (35) имеет вид

$$
\begin{aligned}
\mathfrak{F}_{-\alpha}\left[H_{\alpha}\left(p, \chi_{2}, \chi_{3}\right)\right] & =B^{*} \int d p d \chi_{2} d \chi_{3}\left\langle\chi, p_{2}, p_{3}\left|e^{O^{\dagger}}\right| p, \chi_{2}, \chi_{3}\right\rangle H_{\alpha}\left(p, \chi_{2}, \chi_{3}\right)= \\
& =B^{*} \int d p d \chi_{2} d \chi_{3}\left\langle\chi, p_{2}, p_{3}\left|e^{O^{\dagger}}\right| p, \chi_{2}, \chi_{3}\right\rangle\left\langle p, \chi_{2}, \chi_{3} \mid H\right\rangle= \\
& =\left\langle\chi, p_{2}, p_{3} \mid e^{O^{\dagger}} e^{O} h\right\rangle=\left\langle\chi, p_{2}, p_{3} \mid h\right\rangle=h\left(\chi, p_{2}, p_{3}\right) .
\end{aligned}
$$

Итак, мы определили свертку двух функций в контексте ЗДПФ и вывели соответствующую теорему о свертке, используя представления запутанных состояний и оператор ЗДПФ. Вывод теоремы оказался строгим и кратким благодаря использованию дираковской теории представлений. 


\section{Список литературы}

[1] А. Эйнштейн, Б. Подольский, Н. Розен, УФН, 16:4 (1936), 440-446.

[2] Hong-yi Fan, J. R. Klauder, Phys. Rev. A, 49:2 (1994), 704-707.

[3] Hong-yi Fan, Liang Fu, Internat. J. Theoret. Phys., 44:4 (2005), 529-538.

[4] Hong-yi Fan, Nian-quan Jiang, J. Opt. B Quantum Semiclass. Opt., 5:3 (2003), 283-288.

[5] Hong-yi Fan, Nian-quan Jiang, Chinese Phys. Lett., 19:10 (2002), 1403-1406.

[6] V. Namias, J. Inst. Math. Appl., 25:3 (1980), 241-265.

[7] В. П. Быков, О. О. Силичев, Лазерные резонаторы, Физматлит, М., 2004.

[8] A. C. McBride, F. H. Kerr, IMA J. Appl. Math., 39:2 (1987), 159-175.

[9] D. Mendlovic, H. M. Ozaktas, J. Opt. Soc. Amer. A, 10:9 (1993), 1875-1881.

[10] D. Mendlovic, H. M. Ozaktas, A. W. Lohmann, Appl. Opt., 33:26 (1994), 6188-6193.

[11] H. M. Ozaktas, D. Mendlovic, J. Opt. Soc. Amer. A, 10:12 (1993), 2522-2531.

[12] A. W. Lohmann, J. Opt. Soc. Amer. A, 10:10 (1993), 2181-2186.

[13] J. W. Goodman, Introduction to Fourier Optics, McGraw-Hill, New York, 1968.

[14] L. B. Almeida, IEEE Signal Processing Lett., 4:1 (1997), 15-17.

[15] A. I. Zayed, IEEE Signal Processing Lett., 5:4 (1998), 101-103.

[16] Hong-yi Fan, Yue Fan, Commun. Theor. Phys. (Beijing), 39:4 (2003), 417-420.

[17] F. T. Arrechi, E. Courtens, R. Gilmore, H. Thomas, Phys. Rev. A, 6:6 (1972), 2211-2237.

Поступила в редакцию 7.11.2008, после доработки 14.02.2009 